\title{
Structural Studies on Polyurethane Fibers. I. Crystal and Molecular Structures of Aliphatic Polyurethanes from Hexamethylene Diisocyanate and Some Linear Glycols*
}

\author{
Yasushi SaIto, Seizi NansaI, and Shigetake Kinoshita \\ Department of Fibers and Polymers, Faculty of Technology, \\ Gunma University, Tenjin-cho, Kiryu, Japan.
}

(Received January 4, 1971)

\begin{abstract}
The molecular and crystal structures of aliphatic polyurethane series $-\left[\mathrm{O}-\left(\mathrm{CH}_{2}\right)_{m}-\mathrm{OCONH}-\left(\mathrm{CH}_{2}\right)_{6}-\mathrm{NHCO}-\right]_{p}-$ with $m=2-6$, have been studied by X-ray diffraction methods. Two types of molecular structures have been found. The one ( $m=3,5$ and type II of $m=2$ ) is similiar to the model proposed for $\gamma$-phase of polyamides, where the hydrogen bonding requirements between adjacent chains are satisfied by tilting the urethane group planes approximately $30^{\circ}$ with respect to the fiber axis causing a shortening of the fiber identity periods. The other $(m=4,6$ and type I of $m=2$ ) is similar to the $\alpha$-phase of polyamides, where the molecular chains have planar zigzag conformation. These structures are well confirmed by the comparison of the observed and calculated intensities of X-ray diffraction spots.
\end{abstract}

KEY WORDS Aliphatic Polyurethane / X-ray diffraction / Hydrogen Bonding / Molecular Conformation / Crystal Structure / Crystal Modification /

Linear polyurethanes of aliphatic diisocyanate and linear glycols have the general formula, $-\left[\mathrm{O}-\left(\mathrm{CH}_{2}\right)_{m}-\mathrm{OCONH}-\left(\mathrm{CH}_{2}\right)_{n}-\mathrm{NHCO}-\right]_{p}-$, where $m$ and $n$ are integers and represent the numbers of methylene groups of glycol and diisocyanate, respectively, and the polymers are named $m, n$-polyurethanes. 4, 6-polyurethane (Perlon U) has an X-ray diffraction pattern similiar to that of the unsubstituted polyamides. The chain repeat distance (fiber period) was found to be $19.1 \AA$ by Zahn and Kohler ${ }^{1}$, which is in accordance with that predicted for a fully extended planar zigzag chain. The similarity in the X-ray data between polyamides and polyurethanes suggests that the latter has a conformation of side-by-side zigzag chains with hydrogen bondings between neighbouring chains. This view was pointed out by Borchert ${ }^{2}$, who proposed the two-molecule unit cell, $a=4.95 \AA$, $b$ (fiber axis) $=19.2 \AA, \quad c=8.69 \AA, \alpha=90^{\circ}, \quad \beta=$ $60.2^{\circ}$, and $\gamma=170-176^{\circ}$. Zahn and Winter $^{3}$ reported the four-molecule unit cell, $a=9.55 \AA$,

* This paper has been presented in part at SPSJ 18th Annual Meeting of the Sociaty of Polymer Science, Japan Kyoto, 1969. $b$ (fiber axis $)=19.1 \AA, \quad c=8.40 \AA, \quad \alpha=90^{\circ}, \quad \beta=$ $63-65^{\circ}$ and $\gamma=77^{\circ}$. In their proposed structure the main valences lie in the direction of the fiber axis while hydrogen bondings are extended along $a$-axis and van der Waals forces along $c$-axis.

$m, n$-polyurethanes are classified into four groups by the number of methylene groups in the monomeric units of glycols and diisocyanates, that is, even-even, even-odd, odd-even and odd-odd polyurethanes. When the molecular chains are stretched fully and aligned so as to make hydrogen bondings between adjacent chains, even-even and odd-odd polymers can make all NH.... O hydrogen bonds while evenodd and odd-even polymers can make only $50 \%$ of the NH.... O bonds. In Figure 1, 2, 6and 3,6-polyurethanes are shown schematically and these situations are illustrated. As mentioned below, the infrared work shows that for all linear polyurethanes investigated all the hydrogen bondings are performed, and the structures of the latter groups should be the twisted molecular conformations analogous to those of polyamides ${ }^{4}$. 


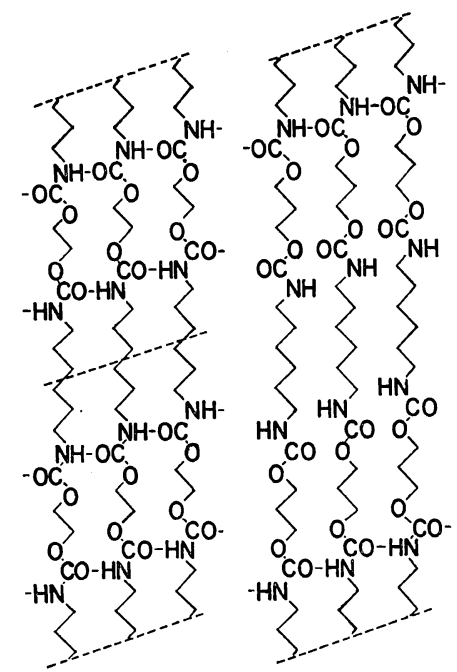

Figure 1. The models with perfect (left, for 2, 6polyurethane) and deficient (right, for 3,6-polyurethane) hydrogen bondings in $m, 6$-polyurethane.

In this paper, the crystal and molecular structures of $m, 6$-polyurethanes were investigated mainly by $X$-ray diffraction studies and are discussed by comparing them with the structures of corresponding polyamides. The series of polyurethanes used in this work were prepared from hexamethylene diisocyanate $(n=6)$ and some linear glycols $(m=2-6)$; that is, poly(ethylene-hexamethylene dicarbamate) for $m=2$, poly(trimethylene-hexamethylene dicarbamate) for $m=3$, poly(tetramethylenehexamethylene dicarbamate) for $m=4$, poly(pentamethylene-hexamethylene dicarbamate) for $m=5$, and poly(hexamethylene-hexamethylene discarbamate) for $m=6$.

\section{EXPERIMENTAL}

\section{Materials}

The polymers were prepared by the direct reaction of the two components ${ }^{5}$. After charging a slight excess (to avoid chain branching and cross-linking) of glycols to the reaction vessel, hexamethylene diisocyanate was added over a period of $\frac{1}{2}$ to $1 \mathrm{hr}$, while gradually increasing the temperature beyond the melting points of each polymer and maintaining them in the vessel until the reaction was completed. Each polymer was spun from the melt, elongated to
200-300\% at a high temperature and then annealed for $15 \mathrm{hr}$ at $125-145^{\circ} \mathrm{C}$ in silicone oil ether under tension or not.

\section{X-ray Diffraction Methods}

The X-ray diffraction patterns were recorded on photofilms using Ni-filtered $\mathrm{CuK}_{\alpha}$ radiation (from Rigaku Denki Co., Ltd. universal X-ray generator). For the purpose of obtaining the X-ray fiber diagrams the substances were examined in the form of monofilaments about $0.4-0.7 \mathrm{~mm}$ in diameter. Standard fiber photographs were taken with the beam perpendicular to fiber axis. In addition, the oscillation photographs were prepared in which the axis of oscillation was perpendicular to the fiber axis. By this means the meridional reflections were brought into reflecting positions. For the intensity estimations, a multiple film technique was applied in clindrical photographs and the intensities of the diffraction spots were measured by visual comparison with the standard intensity scale of the reflections on a separate photo-film and the usual corrections were applied.

\section{Infrared Spectra}

The Infrared absorption spectra in the $800-$ $4000-\mathrm{cm}^{-1}$ region were taken with a Japan Spectroscopic Co. Model DS301 spectrometer. The specimen used was prepared by annealing the powder sample or filament in the same condition as in X-ray diffraction studies and the sample was measured by a $\mathrm{KBr}$ disk.

\section{Melting Points and Densities}

Melting points of these polymers were measured using a polarizing microscope equipped with an electrically heated stage. The temperatures at which the transmitted light vanished were regarded as the melting points.

The density measurements were made by the flotation method, carbon tetrachloride-ethanol mixture being used as the flotation medium. The density of the flotation medium was determined by an Ostwald-type picnometer.

\section{RESULTS AND DISCUSSIONS}

\section{Hydrogen Bondings}

As for the problem of the structures of the linear polyurethane series, the $\mathrm{NH}$.... 
Structural Studies on Polyurethane Fibers. I.

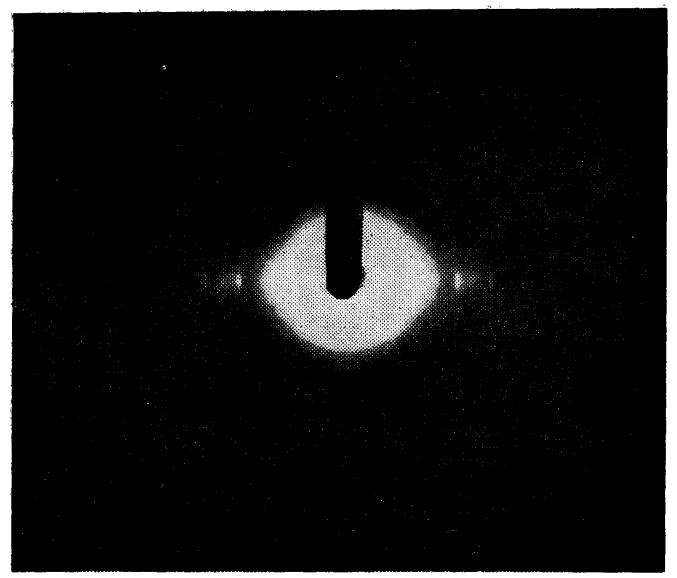

(a)

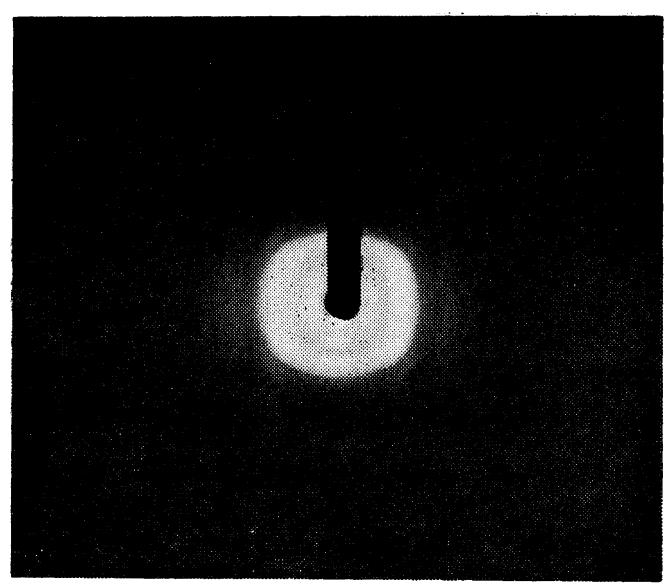

(b)

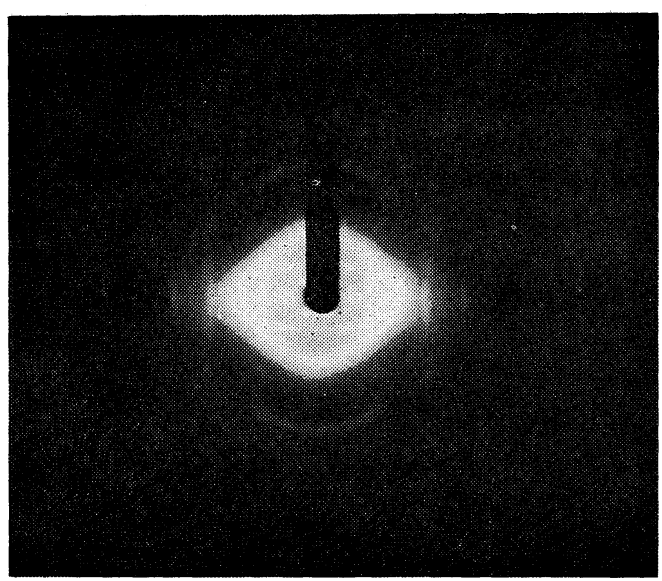

(c)

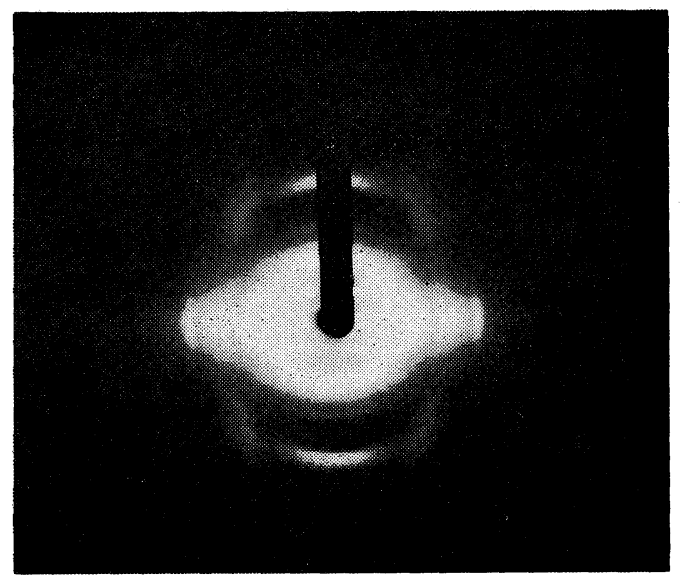

(d)

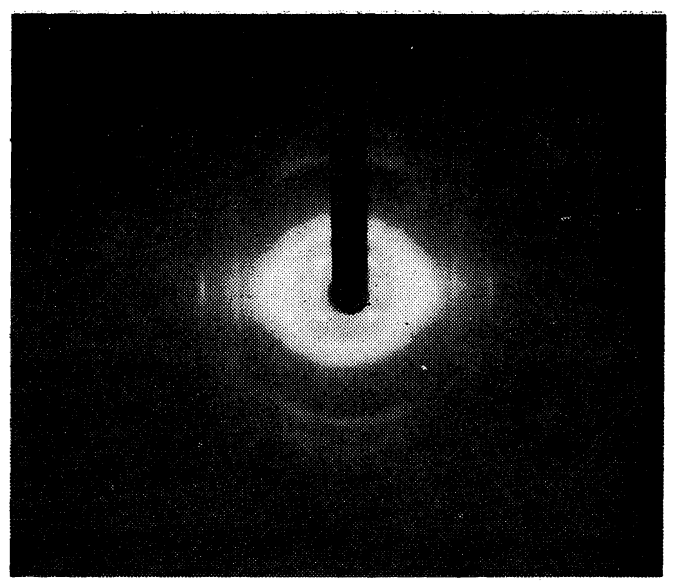

(e)

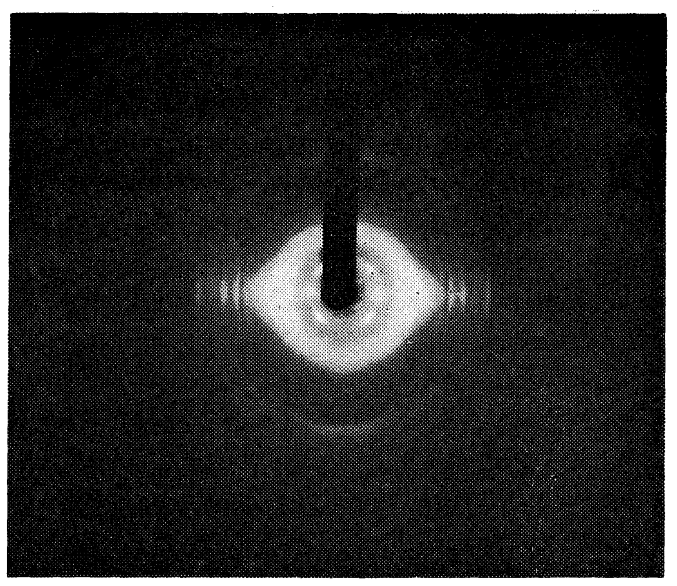

(f)

Figure 2. X-ray diagrams of $m$, 6-polyurethane: (a) 2, 6-(type I), (b) 2, 6-(type II), (c) 3, 6-, (d) 4, 6-, (e) 5,6-, and (f) 6,6-polyurethane. 


\section{Y. Saito, S. Nansai, and S. Kinoshita}

hydrogen bondings formed between urethane groups are particularly important. The basic data on hydrogen bondings in polyurethanes were studied by Tanaka, Yokoyama, and Yamaguchi ${ }^{6}$ by a quantitative infrared spectroscopic study of the model compounds of polyurethanes. They showed that the free $\mathrm{N}-\mathrm{H}$ stretching vibration peak was at $3447 \mathrm{~cm}^{-1}$, and hydrogenbonded $\mathrm{N}-\mathrm{H}$ peak was at near $3300 \mathrm{~cm}^{-1}$.

In the present study, no free $\mathrm{N}-\mathrm{H}$ absorption bands were observable in infrared spectra and no appreciable difference in the absorption range $\left(3300-3500 \mathrm{~cm}^{-1}\right)$ due to the hydrogen bondings existed between the odd and even numbers of $m$ (number of methylene groups from glycols). It may be concluded that in the arrangement of polyurethane chains with odd numbers of $m$, the planar zigzag conformation, which has deficient hydrogen bondings shown in Figure 1, should be modified to satisfy the requirement of perfect performance of hydrogen bondings:

\section{Crystal Structures and Morecular Conformations}

Typical X-ray fiber photographs of $m, n$-polyurethanes with $n=6$ and $m=2-6$ are shown in Figure 2. The diffraction spots of these photographs can be indexed by the lattice parameters of corresponding polymers, shown in Table I, where other crystallographic data, melting points and the calculated fiber periods for planar zigzag conformations are listed. These X-ray patterns are similiar to those of unsubstituted polyamides ${ }^{4}$; $m=2$ (type I), 4 , and 6 to $\alpha$-form, and $m=3$ and 5 to $\gamma$-form. Type II of $m=2$ is analogous to $\gamma$-form. Type I and II of 2, 6polyurethane are prepared by annealing the polymer filament whether under tension or not, respectively. The other polymers have no modifications under these treatments.

In the following descriptions, these polymers are classified conventionally in three groups; (1) 2,6-(type I), 4,6- and 6,6-polyurethanes, (2) 3,6- and 5,6-polyurethanes, and (3) 2,6-polyurethane (type II).

\section{(I) 2,6-(type I), 4, 6- and 6, 6-Polyurethanes}

When the elongated monofilament specimen of 2,6-polyurethane was annealed at $130^{\circ} \mathrm{C}$ under tension, it gave a fiber photograph similar to that of $\alpha$-form of polyamide. This photograph is shown in Figure 2(a), which is different from that of the same 2, 6-polyurethane annealed under no tension (Figure 2(b)), and the former is named type I and the latter type

Table I. Crystallographic data of $m$, 6-polyurethanes

\begin{tabular}{|c|c|c|c|c|c|c|c|c|c|c|c|c|}
\hline \multirow[b]{2}{*}{$m$} & \multirow{2}{*}{$\begin{array}{l}\text { Melting } \\
\text { point, }{ }^{\circ} \mathrm{C}\end{array}$} & \multirow{2}{*}{$\begin{array}{l}\text { Crystal } \\
\text { system }\end{array}$} & \multicolumn{6}{|c|}{ Cell dimensions } & \multirow[b]{2}{*}{$Z^{\mathrm{a}}$} & \multicolumn{2}{|c|}{ Density } & \multirow{2}{*}{$\begin{array}{l}\text { Space } \\
\text { group }\end{array}$} \\
\hline & & & $a, \AA$ & $b, \AA$ & $c, \AA$ & $\alpha, \stackrel{\circ}{\circ}$ & $\beta, \circ$ & $\gamma, \quad \circ$ & & $\begin{array}{l}\text { Calcd, } \\
\mathrm{g} / \mathrm{cm}^{3}\end{array}$ & $\begin{array}{l}\text { Obsd, } \\
\mathrm{g} / \mathrm{cm}^{3}\end{array}$ & \\
\hline $2(\mathrm{II})^{\mathrm{a}}$ & 170 & triclinic & 4.59 & 5.14 & $\begin{array}{l}13.9 \\
(17.03)^{\mathrm{c}}\end{array}$ & 90 & 90 & 119 & 1 & 1.33 & 1.26 & $\mathbf{P} \overline{1}$ \\
\hline$(\mathbf{I})^{\mathrm{b}}$ & 170 & triclinic & 4.93 & 4.58 & $\begin{array}{l}16.8 \\
(17.03)\end{array}$ & 113 & 103 & 109 & 1 & 1.27 & 1.23 & $\mathbf{P} \overline{1}$ \\
\hline 3 & 168 & monoclinic & 4.70 & 8.36 & $\begin{array}{c}33.9 \\
(36.57)\end{array}$ & & & 115 & 4 & 1.33 & 1.20 & $\mathrm{~A} 2 / \mathrm{m}$ \\
\hline 4 & 184 & triclinic & 4.98 & 4.71 & $\begin{array}{l}19.4 \\
(19.54)\end{array}$ & 116 & 105 & 109 & 1 & 1.25 & 1.21 & $\mathbf{P} \overline{1}$ \\
\hline 5 & 158 & monoclinic & 4.70 & 8.36 & $\begin{array}{l}39.0 \\
(41.65)\end{array}$ & & & 115 & 4 & 1.32 & 1.17 & $\mathrm{~A} 2 / \mathrm{m}$ \\
\hline 6 & 165 & triclinic & 5.05 & 4.54 & $\begin{array}{l}21.9 \\
(22.08)\end{array}$ & 112 & 108 & 108 & 1 & 1.23 & 1.16 & $\mathbf{P} \overline{1}$ \\
\hline
\end{tabular}

a Annealed under no tension.

b Annealed under tension.

c The fiber period calculated for planar zigzag conformation assuming bond lengths, $d(\mathrm{C}-\mathrm{C})=1.54 \AA$, $d(\mathrm{C}-\mathrm{N})=1.47 \AA, d(\mathrm{C}-\mathrm{O})=1.43 \AA$ and bond angles $109^{\circ} 28^{\prime}$ for all atoms.

d Number of chemical units per unit cell. 
Structural Studies on Polyurethane Fibers. I.

II. The fiber identity period* for type $\mathrm{I}$ is $16.8 \AA$ and in good agreement with the theoretical value, $17.03 \AA$, calculated for a planer zigzag chain with the accepted bond angles and bond lengths. The observed $d$ spacings could be indexed with a triclinic unit cell as shown in Table I. The density calculated for the proposed unit cell with one chemical repeating unit is $1.27 \mathrm{~g} / \mathrm{cm}^{3}$, while the observed density of this specimen is $1.23 \mathrm{~g} / \mathrm{cm}^{3}$. The slightly higher value of the calculated density suggests that unit cell is acceptable.

If the molecular chains assemble parallel in the crystalline state, all of the NH...O hydrogen bondings can be made. This situation is consistent with the result that the hydrogen bondings are complete by infrared spectroscopy. The chain has centers of symmetry at the middle points of the 4th and 3rd methylene groups in diisocyanate. Hence, the space group of this structure is $\mathbf{P} \overline{1}-\mathrm{Ci}$. Assuming the molecular packing shown in Figure 3, the $\mathrm{NH}$.... hydrogen bonding distances are $2.7 \AA$, and their bonding directions coincide with $b$-axis. The interchain distance perpendicular to hydrogen bondings is $4.2 \AA$, in good agreement with the van der Waals distance between two $\mathrm{CH}_{2}$ groups. The atomic coordinates of the molecules, which describe the proposed structure, are shown in Table II. The comparison between the observed and calculated structure factors is listed in Table III, where for the overlapped reflections the square root of the observed intensities are compared with $\sqrt{m_{i} F_{i}}$ of the calculated structure factors $\left(m_{i}\right.$ : multiplicity of

Table II. Atomic parameters of 2, 6-polyurethane (type I)

\begin{tabular}{crcc}
\hline Atom & \multicolumn{1}{c}{$x$} & $y$ & $z$ \\
\hline $\mathrm{C}$ & 0.0955 & 0.0 & 0.0369 \\
$\mathrm{C}$ & -0.0955 & 0.0 & 0.1108 \\
$\mathrm{C}$ & 0.0955 & 0.0 & 0.1848 \\
$\mathrm{C}$ & 0.0955 & 0.0 & 0.3259 \\
$\mathrm{C}$ & 0.0955 & 0.0 & 0.4630 \\
$\mathrm{~N}$ & -0.0868 & 0.0 & 0.2553 \\
$\mathrm{O}$ & 0.3616 & 0.0 & 0.3259 \\
$\mathrm{O}$ & -0.0816 & 0.0 & 0.3945 \\
\hline
\end{tabular}

* In the description of the crystallographic axis in this study, the fiber axis is always taken to be c-axis.
Table III. Structure factors of 2, 6-polyurethane $\left(\right.$ type I) ${ }^{\mathrm{b}}$

\begin{tabular}{|c|c|c|c|c|c|}
\hline$h k l$ & $\left|F_{\mathrm{cal}}\right|^{\mathrm{c}}$ & $\left|F_{\text {obs }}\right|$ & $h k l$ & $\left|F_{\text {eal }}\right|$ & $\left|F_{\text {obs }}\right|$ \\
\hline 100 & 23.82 & 29.82 & 206 & 0.81 & \multirow{3}{*}{3.52} \\
\hline$\overline{1} 10$ & 21.86 & \multirow{2}{*}{41.82} & 026 & 0.33 & \\
\hline 010 & $35.65)$ & & $\overline{1} 16$ & 2.56 & \\
\hline 110 & 12.84 & \multirow{2}{*}{12.42} & $\overline{2} 26$ & 0.02 & \multirow{4}{*}{1.58} \\
\hline$\overline{2} 10$ & $6.74)$ & & $\overline{2} 16$ & 0.00 & \\
\hline 020 & 13.96 & \multirow{2}{*}{10.49} & 306 & 0.11 & \\
\hline$\overline{2} 20$ & 4.37) & & $0 \overline{1} 6$ & $1.21 /$ & \\
\hline 200 & 5.71 & \multirow{2}{*}{6.58} & 136 & 0.06 & \multirow{4}{*}{1.93} \\
\hline$\overline{1} 20$ & $10.36)$ & & $\overline{3} 26$ & 0.57 & \\
\hline 120 & 4.61 & \multirow{2}{*}{5.03} & $0 \overline{2} 6$ & 1.34 & \\
\hline$\overline{3} 20$ & 0.13 & & $3 \overline{2} 6$ & $0.30^{\prime}$ & \\
\hline 030 & 4.34 & \multirow{2}{*}{4.20} & 107 & 4.50 & \multirow{3}{*}{$\mathrm{s}^{\mathrm{a}}$} \\
\hline$\overline{3} 30$ & $0.04)$ & & 117 & 2.04 & \\
\hline$\overline{1} 11$ & $3.06)$ & \multirow{3}{*}{6.22} & 027 & 0.06 & \\
\hline 011 & 3.74 & & 107 & $2: 12$ & \multirow{2}{*}{6.42} \\
\hline 101 & $3.01 /$ & & 117 & $6.46)$ & \\
\hline 202 & 2.22 & \multirow{2}{*}{1.99} & $\overline{2} 27$ & 3.02 & \multirow{3}{*}{4.38} \\
\hline $2 \overline{12}$ & $2.95)$ & & 217 & 0.94 & \\
\hline$\overline{1} 13$ & 2.50 & 1.50 & 127 & 0.70 & \\
\hline$\overline{2} 13$ & $0.17)$ & \multirow{2}{*}{2.10} & 037 & 0.01 & \multirow{6}{*}{4.05} \\
\hline 023 & $1: 53)$ & & 137 & 0.78 & \\
\hline 123 & 0.20 & \multirow{4}{*}{2.48} & $\overline{2} 27$ & 3.02 & \\
\hline$\overline{2} 03$ & 0.20 & & 307 & 1.20 & \\
\hline $2 \overline{2} 3$ & 0.14 & & $\overline{2} 17$ & 6.51 & \\
\hline $3 \overline{1} 3$ & 0.12 & & $0 \overline{17}$ & $0.41^{\prime}$ & \\
\hline 303 & 0.70 & \multirow{3}{*}{2.03} & 407 & 0.24 & \multirow{3}{*}{4.85} \\
\hline $0 \overline{2} 3$ & 1.20 & & $4 \overline{1} 7$ & 0.48 & \\
\hline$\overline{1} 33$ & 0.36 & & $\overline{3} 27$ & $0.72 /$ & \\
\hline 005 & 1.51 & 1.51 & 008 & 0.82 & \multirow{3}{*}{1.13} \\
\hline$\overline{1} 15$ & $1.47)$ & \multirow{2}{*}{1.97} & $\overline{1} 28$ & 0.09 & \\
\hline 115 & $0.69)$ & & 208 & 0.23 & \\
\hline 016 & 0.82 & $\mathrm{vw}^{\mathrm{a}}$ & & & \\
\hline
\end{tabular}

a Not estimated due to being out of sphere of reflection.

b In Tables 3, 5 and 7, the structure factor of the reflection, which are not observed on the X-ray photographs, are not listed. The calculated values of these reflections have been confirmed to be weaker than those of observed reflections.

c For the over-lapped reflections $\sqrt{m_{i} F_{i}{ }^{2}}$ of the calculated structure factors $\left(m_{i}\right.$ : multiplicity of $i$ th plane) are compared with the square root of the observed intensities. 
$i$ th plane). The bond lengths and bond angles are not changed from the accepted values as shown below Table I. The calculated structure factors have been corrected by uniform temperature factors with $B=9.0 \AA^{2}$. The value of reliability factor $R=\Sigma\left|\left(\left|F_{\text {obs }}\right|-\left|F_{\text {cal }}\right|\right)\right| / \Sigma\left|F_{\text {obs }}\right|$ is $20.2 \%$ for the observed reflections.

Comparisons of the fiber patterns shown in Figure 2(a), (d), and (f) and of the lattice parameters listed in Table $I$, indicate that the 4, 6- and 6,6-polyurethanes have similar molecular and crystal structures to that of 2,6-polyurethane (type $I$ ). The fiber identity periods are $19.4 \AA$ for 4,6 - and $21.9 \AA$ for 6, 6-polyurethane, and are in good agreement with the theoretical values, $19.54 \AA$ and $22.08 \AA$, respectively, calculated for a planar zigzag conformation. The $d$ spacings and relative intensities of equatorial reflections of these polymers are identical with those of 2,6-polyurethane (type I) and the fiber identity periods have larger values which correspond to the excess numbers of methylene groups. These results suggest that the crystal and molecular structures of 4,6- and 6, 6-polyurethanes have the analogous structure as shown in Figure 3, where those fiber periods

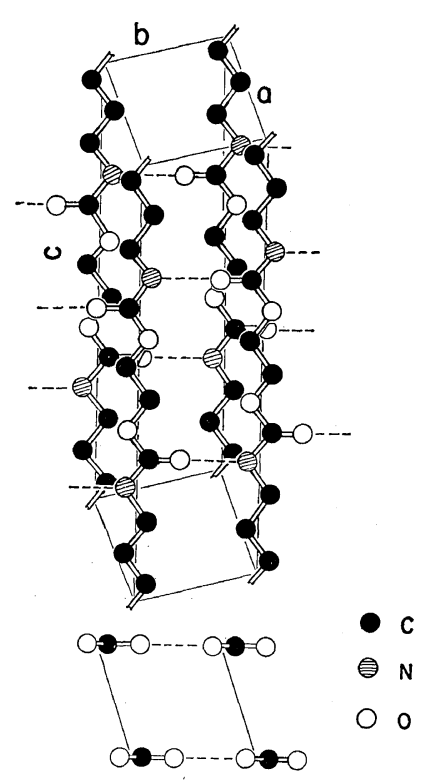

Figure 3. A drawing of the structure of 2,6-polyurethane (type I) viewed perpendicular to the fiber axis (top), and viewed along the fiber axis (bottom). are $19.4 \AA$ and $21.9 \AA$, respectively, and the numbers of methylene groups from glycols are different from that of 2,6-polyurethane.

The crystal and molecular structure of 4,6polyurethane mentioned here is almost the same as the figure given in the report by Zahn and Kohler ${ }^{1}$, except exchanging the $b$ - and $c$-axis. But the unit cell dimensions mentioned in theirs and Brochert's ${ }^{2}$ are not understood in this concept.

\section{(2) 3,6- and 5, 6-Polyurethane}

Figures 2(c) and (e) show the X-ray patterns of 3,6- and 5,6-polyurethane, respectively. These patterns are very similiar to $\gamma$-form of polyamide. The observed $d$ specings can be indexed by monoclinic unit cells as shown in Table I. The fiber indentity periods of these two polymers are shorter than the calculated ones for planar zigzag conformation. The shortenings in these polymers have the same value and are not influenced by the numbers of methylene groups from glycols and may be well justified by the distortion of the chain from a planar zigzag conformation due to the inclination of urethane group planes to the residual methylene group planes. For the structure of polyamide a model is proposed, in which the hydrogen bonding requirements are satisfied by tilting the amide planes approximately $30^{\circ}$ with respect to the fiber axis. ${ }^{7}$ Such a conformation is brought about by rotation around the $\mathrm{C}-\mathrm{C}$ and $\mathrm{C}^{\prime}-\mathrm{N}$ bonds of the groups $\mathrm{C}-\mathrm{C}-\mathrm{C}-\mathrm{C}^{\prime}$ and would obviously cause a shortening of the fiber period. This chain shortening can make the fully hydrogen boundings between the neighbouring chains, which are expected from the results of the infrared spectroscopy.

The molecules in this conformation have two centers of symmetry in the middle points of the 3rd and 4th methylene groups from diisocyanate and two mirror planes in the middle methylene groups from glycols. The unit cells of these polymers have four chemical repeating units, which consist of two molecular chains per unit cell. Systematic absences of X-ray diffraction spots, $k+1 \neq 2 n$ for $h k l, k \neq 2 n$ for $h k 0$ and $1 \neq 2 n$ for 001 , show that the space group these 
structures is $A 2 / m-C_{2 h}^{3}$. In order to satisfy the requirement of full hydrogen bonds (along $b$-axis), the molecule at $(x, y, z)$ should be coupled with one at $(\bar{x}, y+1 / 2, z)$, which is different from the one at $(\bar{x}, \bar{y}+1 / 2, z)$ due to the requirement of symmetry elements of the space group. Hence the crystal requires a statistical disordered structure with respect to the molecular packings, where the molecules exist in both positions at $(x, y, z)$ and $(x, \bar{y}, z)$. The intermolecular spacings between the neighbouring molecules are feasible in the disordered
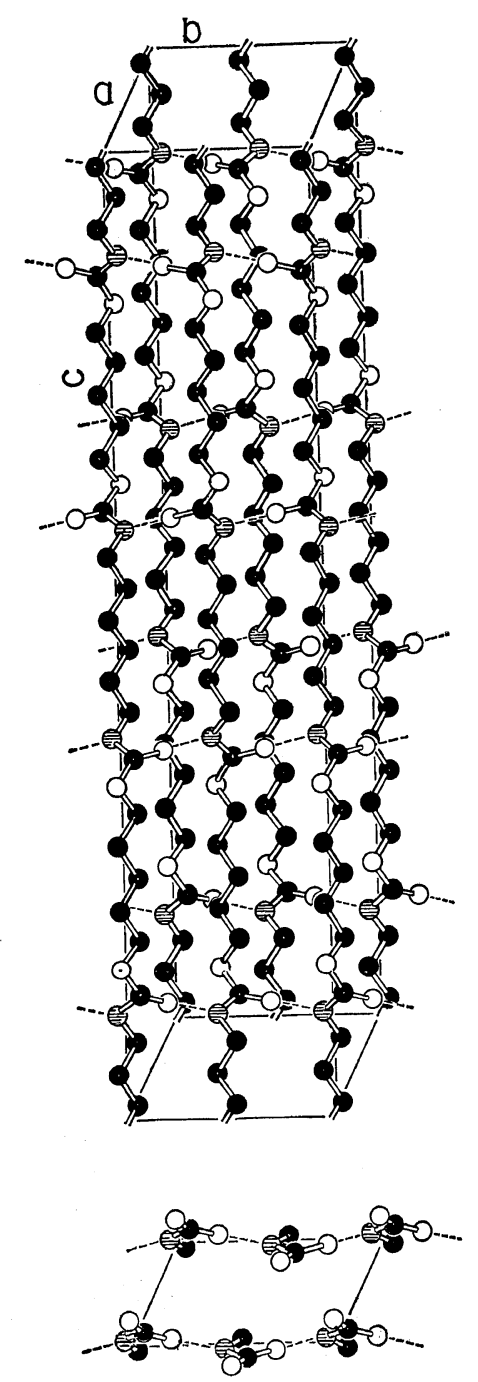

Figure 4. A drawing of the structure of 5,6polyurethane. structure from the viewpoint of the van der Waals distances. This statistical structure is similiar to the case of rubber $^{8}$, in which, however, the statistical structure is not required by the symmetry of the space group. The crystal structure of 5,6-polyurethane is illustrated in Figure 4, at the bottom of which the situation of hydrogen bondings are shown in the cross-section perpendicular to the fiber axis.

The space group may be taken to be $P 2_{1} / m-C_{2 h}^{2}$, where the systematic absences of the spots should be $1 \neq 2 n$ for 001 so as to avoid the statistical structure mentioned above. The space group $A 2 / m-C_{2 \mathrm{~h}}^{3}$ was used for the culculation of the structure factors. Table $\mathrm{V}$ lists the observed and calculated structure factors,

Table IV. Atomic parameters of 5, 6-polyurethanes ${ }^{\mathrm{a}}$

\begin{tabular}{crcc}
\hline Atom & $x$ & $y$ & $z$ \\
\hline $\mathrm{C}$ & -0.1925 & \pm 0.0191 & 0.0156 \\
$\mathrm{C}$ & -0.0093 & $\mp 0.0215$ & 0.0469 \\
$\mathrm{C}$ & -0.1925 & \pm 0.0191 & 0.0784 \\
$\mathrm{C}$ & -0.0140 & $\mp 0.0861$ & 0.1373 \\
$\mathrm{C}$ & 0.1784 & \pm 0.1100 & 0.1916 \\
$\mathrm{C}$ & 0.1690 & \pm 0.0430 & 0.2271 \\
$\mathrm{C}$ & 0.3873 & \pm 0.1387 & 0.2500 \\
$\mathrm{~N}$ & -0.0164 & $\mp 0.0191$ & 0.1083 \\
$\mathrm{O}$ & -0.0234 & \pm 0.0227 & 0.1702 \\
$\mathrm{O}$ & 0.0000 & \pm 0.2344 & 0.1332 \\
\hline
\end{tabular}

a The origin is shifted to $(0,1 / 4,1 / 4)$.

Table V. Structure factors of 5, 6-polyurethane

\begin{tabular}{ccclcc}
\hline$h k l$ & $\left|F_{\text {cal }}\right|$ & $\left|F_{\text {obs }}\right|$ & $h k l$ & $\left|F_{\text {cal }}\right|$ & $\left|F_{\text {obs }}\right|$ \\
\hline 100 & 29.99 & 27.82 & $\overline{1} 33$ & 4.98 & 2.34 \\
020 & 21.50 & & 228 & 1.29 & 1.32 \\
$\overline{1} 20$ & 20.86 & 29.96 & 0210 & 3.02 & \\
120 & 5.75 & 7.28 & $\overline{1} 210$ & 0.75 & 5.46 \\
040 & 8.20 & & 1210 & 0.66 & \\
$\overline{2} 40$ & 6.30 & 9.66 & $\overline{2} 210$ & 2.12 & 3.12 \\
320 & 0.38 & & 2210 & 1.46 & 1.06 \\
060 & 0.73 & 2.42 & 0311 & 2.43 & 2.60 \\
$\overline{3} 60$ & 0.60 & & $\overline{1} 115$ & 3.63 & 7.78 \\
220 & 1.46 & 3.16 & 0315 & 5.13 & 3.46 \\
111 & 14.11 & 14.92 & $\overline{2} 216$ & 2.30 & 1.22 \\
$\overline{1} 13$ & 12.87 & 14.46 & 1216 & 3.27 & 2.64 \\
$\overline{2} 13$ & 6.95 & 3.16 & & & \\
\hline
\end{tabular}


the latter being calculated for the atomic coordinates shown in Table IV and corrected by uniform temperature factors with $B=9.0 \AA^{2}$. The overlapped reflections were treated in the same method as in 2,6-polyurethane (type I), and the reliability factor is $19.2 \%$. On the basis of the similarity of X-ray diffraction patterns and molecular structures between 3,6and 5,6-polyurethane, the structure of the former polymer can be taken to be the same as the latter.

\section{(3) Type II of 2, 6-Polyurethane}

The X-ray fiber photograph of 2,6-polyurethane (type II) is shown in Figure 2(b), which resembles that of $\gamma$-form of polyamide. The observed $d$ spacings can be indexed by numerically monoclinic unit cells, but the symmetry elements in them correspond to the triclinic lattice. The observed fiber period is 13.9 $\AA$, which is shorter than that of a planar zigzag conformation based on the generally accepted bond lengths and bond angles for one repeating unit. The difference of the observed and calculated period is $3.1 \AA$ per two urethane groups, which is about twice of 3,6- or 5,6polyurethane. Therefore, in this case, the

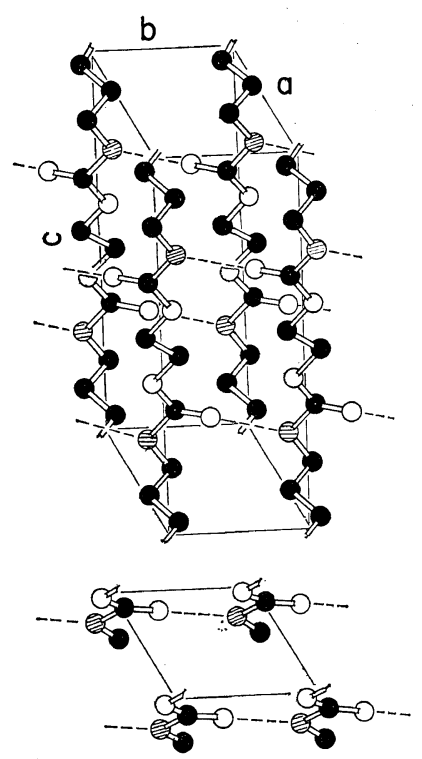

Figure 5. A drawing of the structure of 2,6-polyurethane (type II). methylene groups should be inclined to the fiber axis, while, in the case of 3, 6-polyurethane, the methylene group planes are parallel to the fiber axis. This large shortening is brought about by tilting two methylene group planes from diisocyanate and glycol and two urethane group planes approximately $30^{\circ}$ with respect to the fiber axis. These tiltings are derived by the rotation of $\mathrm{O}-\mathrm{C}$ and $\mathrm{N}-\mathrm{C}^{\prime}$ single bonds about $40^{\circ}$ from trans position in the opposite sites. The molecules have two centers of symmetry

Table VI. Atomic parameters of 2, 6-polyurethane (type II)

\begin{tabular}{crrc}
\hline Atom & $x$ & \multicolumn{1}{c}{$y$} & \multicolumn{1}{c}{$z$} \\
\hline $\mathrm{C}$ & -0.0912 & 0.0828 & 0.0364 \\
$\mathrm{C}$ & -0.2313 & -0.1285 & 0.1094 \\
$\mathrm{C}$ & -0.4248 & 0.0392 & 0.1820 \\
$\mathrm{C}$ & -0.1579 & 0.0719 & 0.3302 \\
$\mathrm{C}$ & -0.1045 & 0.1133 & 0.4741 \\
$\mathrm{~N}$ & -0.2669 & -0.1264 & 0.2561 \\
$\mathrm{O}$ & -0.0044 & -0.0828 & 0.4022 \\
$\mathrm{O}$ & -0.1957 & 0.3312 & 0.3036 \\
\hline
\end{tabular}

Table VII. Structure factors of 2, 6-polyurethane (type II)

\begin{tabular}{|c|c|c|c|c|c|}
\hline$h k l$ & $\left|F_{\text {cal }}\right|$ & $\left|F_{\text {obs }}\right|$ & $h k l$ & $\left|F_{\text {cal }}\right|$ & $\left|F_{\text {obs }}\right|$ \\
\hline 100 & 27.24 & 32.40 & 101 & 11.66 & \multirow{3}{*}{18.0} \\
\hline 010 & 46.24 & \multirow{2}{*}{49.24} & $\overline{1} 11$ & 13.56 & \\
\hline$\overline{1} 10$ & $16.16)$ & & 011 & 0.70 & \\
\hline 110 & 9.81 & \multirow{2}{*}{10.87} & 102 & 7.12 & 9.79 \\
\hline$\overline{2} 10$ & $5.41)$ & & $\overline{1} 12$ & 5.67 & \multirow{2}{*}{9.03} \\
\hline 200 & $4.44)$ & \multirow{2}{*}{8.69} & 012 & 6.79) & \\
\hline$\overline{1} 20$ & 2.59) & & $\overline{2} 12$ & 4.39 & \multirow{3}{*}{2.42} \\
\hline 020 & $7.08)$ & \multirow{2}{*}{7.07} & 112 & $6.79)$ & \\
\hline$\overline{2} 20$ & $7.26)$ & & 103 & 5.18 & \\
\hline$\overline{3} 10$ & 1.38 & - & 013 & 1.66 & \multirow[t]{2}{*}{5.52} \\
\hline 210 & $2.51 \backslash$ & \multirow{4}{*}{4.16} & 113 & $3.27)$ & \\
\hline 120 & 1.16 & & 014 & 0.77 & \multirow{3}{*}{6.55} \\
\hline$\overline{3} 20$ & $1.32)$ & & $\overline{1} 14$ & 1.19 & \\
\hline 300 & 2.33 & & 104 & 5.84/ & \\
\hline$\overline{2} 30$ & 1.13 & \multirow[t]{2}{*}{3.44} & 115 & 1.56 & \multirow{2}{*}{5.30} \\
\hline 130 & $0.69 /$ & & $\overline{2} 15$ & $2.66)$ & \\
\hline 310 & $0.38>$ & \multirow{4}{*}{2.89} & 205 & 6.81 & \multirow{2}{*}{4.12} \\
\hline 220 & 1.70 & & $\overline{125}$ & 0.70 & \\
\hline \multirow[t]{2}{*}{$\overline{4} 10$} & 0.34 ) & & 016 & 0.74 & \multirow{2}{*}{4.92} \\
\hline & & & $\overline{1} 16$ & 1.85 & \\
\hline
\end{tabular}


on the mid-points of $3 \mathrm{rd}$ and 4th methylene groups from diisocyanate and of two methylene groups from glycol. This model allows complete hydrogen bondings between adjacent molecules about $2.7 \AA$ in length.

The proposed model is shown in Figure 5, where the hydrogen bondings lie along the $b$-axis and the interchain distance along the direction perpendicular to $b$-axis is $4.0 \AA$, in good agreement with the van der Waals distance between two metylene groups. Assuming that the space group is $\mathrm{P} \overline{1}-\mathrm{Ci}$ and that the atomic coordinates are given as shown in Table VI the observed and calculated structure factors are listed in Table VII, where the calculated ones are corrected by uniform temperature factors with $B=9.0 \AA^{2}$. In this case, the reliability factor $R$ is $20.2 \%$ for all observed reflections.

Structural Modifications and Comparison with Corresponding Polyamides

For 2, 6-polyurethane, there are two kinds of modification in crystal structure and molecular conformation. For other polymers studied here, no modifications are observed hitherto, even if the same treatment as in 2,6-polyurethane is applied. Especially, for 4,6- and 6,6-polyurethanes, it seems that there is equal probability to be changed in structure. This difference is supposed to occur for the reason that, in 2, 6polyurethane, the molecule may be easily distorted due to the largely different intervals of hydrogen bondings along the fiber axis. However, modifications for the other polymers would be found by different treatments.
The chemical structure of $m, n$-polyurethane, $-\left[\mathrm{O}-\left(\mathrm{CH}_{2}\right)_{m}-\mathrm{OCONH}-\left(\mathrm{CH}_{2}\right)_{n}-\mathrm{NHCO}-\right]_{p}-$, resembles that of $m, n$-polyamide, $-[-\mathrm{CO}$. $\left.(\mathrm{CH})_{m-2}-\mathrm{CONH}\left(\mathrm{CH}_{2}\right)_{n}-\mathrm{NH}\right]_{p}$-, while the oxygen atom in the urethane group is exchanged by the methylene group adjacent to the amide group. This is the reason that the structure of these two series are analogous and that present study has been accompli hed with the aid of the results reported on the structures of polyamide. In this study, there has been no appreciable difference between structures of these two series. But, it seems that the influence of the structural difference between amide and urethane groups on their crystal structures would be apparent by more detail investigations, especially, for the structures of the members with the lesser number of $m$ or $n$ of polyurethane, which we are investigating now.

\section{REFERENCES}

1. H. Zahn and K. Kohler, Kolloid Z., 118, 115 (1950).

2. W. Z. Borchert, Angew. Chem., 63, 31 (1951).

3. H. Zahn and V. Winter, Kolloid Z., 128, 142 (1952).

4. Y. Kinoshita, Makromol. Chem., 33, 1 (1959); D. C. Vogelsong, J. Polym. Sci., Part A, 1, 1055 (1963).

5. C. S. Marvel and J. H. Johnson, J. Am. Chem. Soc., 72, 1674 (1950).

6. T. Tanaka, T. Yokoyama, and Y. Yamaguchi, J. Polym. Sci., Part A-1, 6, 2137 (1968).

7. Y. Kinoshita, Makromol. Chem., 33, 21 (1959).

8. S. C. Nyburg, Acta Cryst., 7, 385 (1954). 